\title{
Consulting the Internet: Using Online Search Engines and Its Impact on the Practice of Medicine in the $21^{\text {st }}$ Century
}

\author{
Cheng Chris-Tin ${ }^{1}$, Fatimah Lateef ${ }^{2}$ \\ ${ }^{1}$ Monash University, Australia ${ }^{2}$ Department of Emergency Medicine, Singapore General Hospital, Singapore.
}

\begin{tabular}{ll}
\hline ARTICLE INFO \\
Received & $: 09 / 03 / 2014$ \\
Accepted & $: 11 / 05 / 2014$ \\
Published & $: 01 / 09 / 2014$ \\
\hline
\end{tabular}

\section{KEYWORD}

Internet

Search engines

Diagnosis

Patient-doctor relationship

\section{ABSTRACT}

The Internet has become an ever-expanding treasure trove of health information, which is easily accessible and mobile, for both healthcare personnel and laypersons. An Internet search is clearly a trendy approach to seek knowledge, especially at the point of care. As more information is available, both doctors and patients are able to use the information to their advantage - doctors to augment diagnoses and learning, and patients, to be more informed about their conditions. However, as healthcare personnel require information that is up-to-date, reliable and applicable to the situation, the reliability of information found online could be called into question, although studies seem to have found it to be largely accurate. Furthermore, patients may not always have the intended success with internet searches, and this can result in wrong diagnoses, over-diagnoses, missed symptoms, patient denial, and the undermining of the doctor-patient relationship. In conclusion, it seems that modern doctors of the $21^{\text {st }}$ century should and need to be tech-savvy, especially in terms of the Internet resources available to them. In addition, they should realize that patients may now assume a more active role in their health decision-making process, and therefore make the appropriate adjustments in terms of the evolving doctor-patient relationship, in a new world order which is moving rapidly towards ubiquitous connectivity.

(C) Medical Education Department, School of Medical Sciences, Universiti Sains Malaysia. All rights reserved.

CORRESPONDING AUTHOR: Assoc Prof Fatimah Lateef, Dept of Emergency Medicine, Singapore general Hospital, Outram Road, Singapore 169608. E-mail: fatimah.abd.lateef@sgh.com.sg

\section{Introduction}

Since the dawn of the "dot com" age, the Internet has become an ever-expanding treasure trove of information. This information is easily accessible, mobile, and ubiquitous, easily available to any citizen of the world with just a click on their computer or mobile device. The real revolution may have started with the implementation of search engines that enables us to quickly retrieve the 'exploding' amount of information in a resourceful way, but at the same time focus on our relevant clinical questions while filtering the evidence.

A survey done in 2012 by the Pew Internet \& American Life Project (1) interviewed 3014 adults and found that $59 \%$ of American adults go online in search of health information, with $77 \%$ beginning their search at a search engine. Undeniably, the Internet provides unrestricted access to a sea of information, and information is indeed power. Thirty-five percent of adults have used the Internet to figure out what medical 
condition they have had. Disconcertingly, a similar report by the same institution in 2006 (2) showed that a staggering $75 \%$ of users do not consistently check the source and date of the health information they find online. Nevertheless, the same study did find that successful health information searches may bolster health seekers' confidence, with $31 \%$ of health seekers saying they have been significantly helped following information or advice found on the Internet.

It is clear that the Internet is a force to be reckoned with, even from the doctor's point of view. The New England Journal of Medicine raises the example of a case conference involving a diagnostic dilemma that had stumped even a distinguished visiting professor, which was resolved when a fellow made the stunning diagnosis of IPEX (immunodeficiency, polyendocrinopathy, enteropathy, X-linked) in an infant. As reported, she had Googled the salient features of the case, and the answer "popped right up" (3).

Indeed, the ease and mobility at which information can be assessed with swift keystrokes on a device connected to the Internet (be it a computer, Ipad, or a smartphone) can be astounding. Numerous applications and broadband services have made it possible to access information for health care at the point of care. Consequently, could Internet search engines possibly help to guide doctors, as well as patients, to the right answers? Or conversely, could this information be the bane of every physician's existence?

\section{Milking the Internet for Information}

As medical practitioners, we are trained to analyse a large amount of information, organise it systematically, and then synthesise the relevant material into a likely diagnosis, which would then guide treatment. However, what if a simple Google search could facilitate this complicated thought process?

A pilot study conducted in 2009 with a trainee doctor and two final year medical students showed the potential for Internet search engines. In this study, the participants were asked to determine the diagnosis for twenty-six cases presented in the case records from the New England Journal of Medicine. The use of Google and PubMed was found to have increased accurate diagnoses by approximately $10 \%$, and could therefore substantially aid trainees in the differential diagnosis process (4). A similar study was again conducted in 2010 when five medical students from the International Medical University, Malaysia utilizing the Google search engine to diagnose cases also derived from the New England Journal of Medicine, achieved a congruency rate of $44.7 \%$. This was deemed commendable in light of the case complexity and the inexperience of the students (5).

Therefore, there is definitely evidence that armed with Google, it is indeed possible for Internet search engines to augment the thought processes leading to diagnosis, especially with regard to trainee doctors and students.

Furthermore, as "Dr. Google" becomes an increasingly common household name, it is important to remember that the Internet is not only expanding physicians' horizons, but also impacting the average layperson as well. It is no longer uncommon for patients to search for their own symptoms, and self-diagnose themselves with whatever seems to be the most accurate disease that pops up. In his paper "Googling for a diagnosis - use of Google as a diagnostic aid" (6), Tang et al describes an anecdote from their own experiences whereby the father of a 16 year old water polo player presenting with acute subclavian vein thrombosis, upon being told that the diagnosis was uncertain, accurately diagnosed Paget-von-Schrötter syndrome on his child. The father had Googled his son's symptoms, and could even provide the treating team with the pathophysiology of the condition. In fact, the Pew study (1) found that health seekers who attempted a diagnosis were more likely to have it confirmed rather than rejected by a doctor, i.e. $41 \%$ vs. $18 \%$.

In addition to aiding the diagnostic process, going online following a visit to the doctor's 
office can be beneficial as well - a patient can verify a diagnosis, expand on their understanding of their ailment, or even participate in online support groups. In fact, Fox (7) has found that although health professionals are rated as health seekers' top source for technical questions such as diagnosis and treatment, nonprofessionals like friends and family are rated higher for emotional support and quick remedies. In fact, there is evidence that people are interested in connecting with and learning from each other, with $21 \%$ of American adults saying they have turned to others with similar health conditions. Therefore, this study concludes that "clinicians might do well to look into online patient communities and consider recommending them as resources for patients" where appropriate.

\section{When the Internet turns on us}

While online searches can lead to accurate diagnoses that even the most experienced physician can miss, the flip side is a flurry of unnecessary anxiety and distress, such as the "tension headache" that "must be a brain tumour". Indeed, plumbing the depths of the internet for diagnoses and explanation for ailments have led to what some refer to as "cyberchondria", a term describing those who believe they have a disease that they read about online. Self-diagnosis is not always beneficial, as Pillay highlighted in his article "The Dangers of Self Diagnosis". He argues that self-diagnosis is a recipe for disaster; explaining possibilities of wrong diagnoses, over-diagnoses, missed symptoms, patient denial, and the undermining of the doctor-patient relationship (8).

In 2009, Schembri et al (9) found that, out of a group of 223 symptomatic patients attending a genitourinary clinic in the United Kingdom, $45.3 \%$ looked up their symptoms on the internet, with no significant difference in the ages of those who looked up their symptoms or not. $90.1 \%$ of the patients used a Google search engine as the starting point for their search, highlighting the pervasiveness of this popular search engine. However, only $13.9 \%$ of the patients who looked up their own symptoms made the correct diagnosis, which strongly echoed the findings of a Swiss study involving 4 non-physicians and 4 physicians. This paper revealed that the average diagnostic success arrived at by non-physicians using Google was $21 \%$ (95\% CI $4.5-9.7 \%$ ), which though commendable, was significantly lower than that of the physician's score $(50.9 \%$, 95\% CI 37.4-64.5\%)(10).

Therefore, it seems clear that though nonphysicians may occasionally reach the correct diagnosis using Internet search engines, "Dr. Google" has not yet usurped the role of the physician.

Yan \& Sengupta (11) from the Hong Kong University of Science and Technology examined the information that influenced health seekers' decisions on whether they had a disease or not, looking also at base rate (disease prevalence in a population) and case information (an individual's disease symptoms). They developed a theory that the importance a health seeker places on base rate and case information depends on the affected person's "psychological distance" to them (self being the closest, a stranger being very distant). This meant that while assessing themselves, health seekers would place a greater importance on case information (their symptoms). Conversely, when assessing a stranger, base rate (how prevalent an illness is in that population) would play a greater role. Therefore, they concluded "such symptommatching exercises may lead consumers to overestimate the likelihood of getting a serious disease because they focus on their symptoms while ignoring the very low likelihood that their symptoms are related to any serious illness".

Therefore, despite the vast amounts of information available, one must still be able to sift through the top links and identify reliable sources as well as relevant information to correctly arrive at the correct diagnosis. Here lies the distinction between the medically-trained professional and a non-physician - the use of the Internet as a diagnostic tool can be a doubleedged sword, assisting the physician in making decisions, but also arming the patients and their families with unwarranted worry. In addition, as Lombardi et al pointed out, the effectiveness of 
an internet search can be attributed to several variables, including internet skills, computer skills, and the researcher's ability to solve clinical problems (12).

It is imperative that doctors realize that patients may now assume a more active role in their health decision-making process, and therefore make the appropriate adjustments in terms of the evolving doctor-patient relationship. According to Lindberg \& Humphreys (13), as access to electronic information becomes more pervasive, there are more risks associated with "inefficient or incomplete retrieval of the available evidence". Therefore, medical practitioners need to play the corrective role and become the gatekeepers - either to assuage fears of the hypochondriac, or to take into account the opinion of the accurate Googler.

\section{Is this a reliable source?}

As physicians, the quality and reliability of information gleaned from various sources is paramount to providing quality medical treatment. Healthcare professionals require information that is up-to-date, reliable, and applicable to local situations. In fact, a 2006 study presented at the American Medical Informatics Association annual symposium showed that $92 \%$ of physicians access a targeted site rather than utilize a search engine (such as Google) to gather medical information (14), revealing the healthy skepticism of the reliability of the average search engine to provide accurate information. However, such targeted websites usually require a paid subscription, and generally do not allow free access by the public. Therefore, skeptical or not, one must consider the quality of information available to the general public. As mentioned above, $75 \%$ of users do not consistently examine the quality of the information they find online. Poor information and underdeveloped health literacy skills can lead many patients to have false beliefs, ranging from instant weight loss to cures for incurable diseases.

A study by the IMS Health Institute in the United States found that Wikipedia is the single leading source of medical information for patients and healthcare professionals, with the top 100 Wikipedia pages for healthcare topics being accessed on average of 1.9 million times in 2013 (15). According to the University of California, San Francisco's Professor of Psychiatry, Dr. Amin Azzam, there is "a lot of room for improving the quality of Wikipedia in the medical domain because doctors are late-comers to the resource". In addition, he admits that all his medical students use Wikipedia first, as it is "written in a way that they understand"(16). Therefore, it is likely that regardless of accuracy, websites like Wikipedia will appeal to the less learned of the profession as well as the average layperson because of its simplicity.

Google's scientific offspring, Google Scholar, provides more links to scientific journals and peer-reviewed articles while retaining the simplicity of a Google search, leading to its growing popularity with medical students and doctors. Nevertheless, as Guistini (17) critiques it, "What does Google consider 'scholarly'?", questioning the reliability of the search algorithm for keeping abreast of the constantly updating scientific literature.

Nevertheless, despite the internet's prevailing reputation for unsubstantiated sources and the lack of peer reviewed articles, in 2006, Tang \& $\mathrm{Ng}$ showed Google's usefulness by utilizing it to derive differential diagnoses for cases from the New England Journal of Medicine, which revealed the correct diagnosis in $58 \%$ of cases (6). However, it did find that "searches are less likely to be successful in complex diseases with non-specific symptoms, or common diseases with rare presentations. Similarly, 2009 saw Lombardi et al prove that Google was useful in identifying appropriate diagnoses in complex immunological and allergic cases, with their three investigators making an average of $72.6 \%$ correct diagnoses(12), further reinforcing the notion that Google could potentially be a reliable source. 


\section{Conclusion}

Doctors of today will have to gain, assess, apply and integrate new knowledge in real time. The Internet is a valuable tool that can offer this. Internet search engines broaden the possible diagnoses and allow the medical practitioner to consider diagnoses that he may not have previously thought of. As such, Internet resources enable physicians to gather evidence promptly, saving time and obtaining up-to-date information. They generally seem to be more useful in assisting rare diagnoses where highly specialized knowledge would be required to make a diagnosis. An important parallel could be made in the surgical field, where robotic surgery is gradually making inroads in assisting surgeons to perform difficult surgical techniques (18).

Now is the age of a growing population of patients who are equipped and empowered with information, who want to be engaged in their own healthcare. According to the Pew Research Centre, "experts foresee an ambient information environment where accessing the Internet will be effortless and people will tap into it so easily it will flow through their lives 'like electricity."(19) As such, it seems that modern doctors of the $21^{\text {st }}$ century should and need to be tech-savvy, especially in terms of the Internet resources available to them. When properly used, search engines can lead to faster and possibly more accurate diagnoses, aiding physicians and patients alike in furthering the efficiency and effectiveness of medical treatment in today's society. $(19,20)$. The doctors of today will have to adapt appropriately to the integration of internet into our daily lives as well as into the practice of Medicine.

\section{Reference}

1. Fox S, Duggan M. Health Online 2013. Washington, D.C: Pew Internet \& American Life Project, 2013.

2. Fox S. Online Health Search 2006. Washington, D.C: Pew Internet \& American Life Project, 2006.
3. Greenwald R. And a diagnostic test was performed. New England Journal of Medicine. 2005;353:2089-90.

4. Falagas M, Ntziora F, Makris G, Malietzis G, Rafailidis P. Do PubMed and Google searches help medical students and young doctors reach the correct diagnosis? A pilot study. European Journal of Internal Medicine. 2009;20(8):788-90.

5. Nalliah S, Chan S, Ong C, Suthan T, Tan K, She $\mathrm{V}$, et al. Effectiveness of the use of internet search by third year medical students to establish a clinical diagnosis. Singapore Medical Journal. 2010;51(4):3328.

6. Tang H, Ng J. Googling for a diagnosis - use of Google as a diagnostic aid: internet based study. British Medical Journal. 2006;333(7579):1143-5.

7. Fox S. After Dr Google: peer-to-peer health care. Pediatrics. 2013;131(Suppl 4):S224-5.

8. Pillay S. Debunking myths of the mind. Psychology Today; 2010 [cited 201413 Feb]; Available from: http://www.psychologytoday.com/blog/debu nking-myths-the-mind/201005/the-dangersself-diagnosis.

9. Schembri G, Schober P. The internet as a diagnositc aid: The patients' perspective. International Journal of STD and AIDS. 2009;20(4):231-3.

10. Siempos I, Spanos A, Issaris E, Rafailidis $P$, Falagas M. Non-physicians may reach correct diagnoses by using Google: A pilot study. Swiss Medical Weekly. 2008;138(4950):741-5.

11. Yan D, Sengupta J. The influence of base rate and case information on health-risk perceptions: A unified model of selfpositivity and self-negativity. Journal of Consumer Research. 2013;39(5):931-46.

12. Lombardi C, Griffiths E, McLeod B, Caviglia A, Penagos M. Search engines as a diagnostic tool in difficult immunological and allergologic cases: Is Google useful? Internal Medicine Journal. 2009;39(7):45964.

13. Lindberg D, Humphreys B. 2015 - The future of medical libraries. New England Journal of Medicine. 2005;352(11):1067. 
14. Leo G, LeRouge C, Ceriani C, Niederman F. Websites most frequently used by physician for gathering medical information. AMIA Annual Symposium Proceedings Archive. 2006:902.

15. IMS Health: Pharma should make better use of social media to engage patients and improve the use of medicines. IMS Health Institute, 2013.

16. Dr. Wikipedia: The 'Double-Edged Sword' of crowdsourced medicine. National Public Radio. 8 Feb 2014.

17. Giustini D. How Google is changing medicine. British Medical Journal. 2005;331(7531):1487-8.

18. Guru K, Kuvshinoff B, Pavlov-Shapiro S, Bienko M, Aftab M, Brady W, et al. Impact of robotics and laparoscopy on surgical skills: A comparative study. Journal of the American College of Surgeons. 2007;204(1):96-101.

19. Anderson J, Lee R. Digital Life in 2025. USA: Pew Research Centre; 2014 [updated 11 Mar 2014; cited 16 Apr 2014]; Available from:

http://www.pewinternet.org/2014/03/11/digit al-life-in-2025/. 